\section{Comme sur un terrain de baby-foot ...}

\author{
M. Kondo Oestreicher
}

L'initiative populaire fédérale intitulée pour une meilleure maîtrise des primes de l'assurance - maladie, présentée par le rassemblement des assurés et des soignants constitué dans ce but, mérite votre soutien.

Le thème socio-politique dominant est que notre système sanitaire est menacé et que la maîtrise des dépenses en santé est urgente. A force d'observer les mouvements des idées sur le sujet, attribuant les responsabilités tantôt aux uns, tantôt aux autres acteurs du système de santé, c'est un peu comme si nous suivions des yeux un ballon de football. Nous sommes en effet les joueurs d'une équipe, solidairement entravés par des règles de jeux comme le sont les joueurs d'un baby-foot. Les mêmes contraintes qui s'exercent sur le manche nous agitent. Et la marge de manœuvre dont nous disposons est réduite. Le principe du jeu, simplifié pour les besoins de l'argumentation, est que

Correspondance:

Dr Mitsuko Kondo Oestreicher

HUG

Pharmacologie clinique

Rue Micheli-du-Crest 24

CH-1211 Genève 14 le déséquilibre des forces finira par faire entrer bon gré mal gré la balle dans le but. Les joueurs doivent donc imprimer des chiquenaudes autant que possible pour garder la balle en mouvement. L'initiative populaire fédérale intitulée pour une meilleure maîtrise des primes de l'assurance maladie en est une. Elle sera présentée à la Chambre Médicale ce 13 décembre 2001 et sera soumise à la collecte des signatures prochainement. Bien que les relations de l'ASMAG avec le comité du RAS ne soit pas sans quelques difficultés, nous pouvons contribuer à sa diffusion, parce que l'initiative est bonne. Elle réclame une adaptation de l'application de la LAMal, en particulier en matière de contrôle des assureurs - maladie et du calcul des primes, notamment en séparant dans leurs bilans et leurs comptes d'exploitation l'assurance obligatoire des soins des autres domaines de l'assurance-maladie. Elle vise à une répartition contrôlée des compensations de risque par un Fonds nouvellement créé, composé de représentants de tous les acteurs du système de santé, les assureurs, les instances politiques et aussi les patients et les soignants. Cela ne peut être atteint que par un nouvel article constitutionnel (art. 117 al. 3), ce qui donne une forme élaborée du texte de l'initiative pour assurer ses buts fondamentaux. Mais les principes en sont simples et le corps médical bénéficie d'une occasion favorable de la diffuser solidairement avec les assurés, dont on conviendra que certains sont aussi des patients dont il ne fait aucun doute que nous sommes solidaires. Cette initiative est une impulsion puissante qui a le potentiel de contribuer au but recherché: la maîtrise des dépenses en santé. Nous sommes directement concernés, nous mobiliserons-nous?

Pour en savoir en plus: http://www.rassemblement.ch/ accueilRAS.html 